\title{
Popliteal Venous Pseudoaneurysm and Arteriovenous Fistula after Orthopedic Surgery
}

\author{
Bertrand Saint-Lèbes $^{1 *}$, Emilie Chastonay ${ }^{1}$, Olivier Borens ${ }^{2}$, Céline Dubuis $^{1}$, Sébastien Déglise ${ }^{1}$, \\ François Saucy ${ }^{1}$, Hervé Rousseau ${ }^{3}$, Jean-Marc Corpataux ${ }^{1}$ \\ ${ }^{1}$ Department of Thoracic and Vascular Surgery, Lausanne University Hospital, Lausanne, Switzerland \\ ${ }^{2}$ Department of Orthopaedic and Trauma Surgery, Lausanne University Hospital, Lausanne, Switzerland \\ ${ }^{3}$ Department of Vascular Interventional Radiology, University Hospital, Toulouse, France \\ Email: *saintlebes.b@me.com
}

Received December 6, 2012; revised January 12, 2013; accepted January 25, 2013

\begin{abstract}
Arthroscopically assisted reconstruction of the anterior cruciate ligament (LCA) is generally a safe and effective procedure with a low rate of vascular complications. We report on a case of a 33-year-old woman with a combined arteriovenous fistula (AVF) and venous popliteal aneurysm that developed 6 months after arthroscopic anterior cruciate ligament reconstruction. At clinical exam the patient presented with left popliteal and calf pain, a tender pulsatile mass posterior to her left knee, popliteal bruit and a thrill at the popliteal fossa. CT scan showed an AVF arising from the left popliteal artery. An aneurysm was seen to fill at the same time as the popliteal vein. Findings at open surgical revision included AVF between the tibioperoneal trunk and the popliteal vein and a venous popliteal aneurysm at the level of the arteriovenous communication. The aneurysm and fistula were repaired. The patient had an uneventful follow up with complete recovery. We also report an endovascular treatment of a iatrogenic arterio-venous fistula.
\end{abstract}

Keywords: Arthroscopy; Complication; Arteriovenous Fistula; Anterior Cruciate Ligament; Venous Aneurysm

\section{Introduction}

Arthroscopy of the knee, the most commonly performed orthopaedic procedure, has become a very effective and safe procedure when performed by experienced arthroscopists [1,2]. Conversely, the actual incidence of complications from arthroscopic knee surgery is rather low. The overall complications rate in knee arthroscopy ranges from $0.56 \%$ to $8.2 \%$ [3]. Vascular complications are reported to occur even more rarely, in less than 0.005 per cent of cases [3], with the majority of these injuries to the popliteal artery and/or vein. Aneurysms following arthroscopic procedures including meniscectomy and meniscal repair have also been described. Popliteal artery injury following arthroscopic ACL reconstruction is quite rare [4-6]. The cause of arterial lesions in ACL surgery is mostly related to the type of surgery and the method of graft fixation. There are few published studies reporting the occurrence of popliteal artery injury during arthroscopic ACL reconstruction [7,8]. AVF is thus an uncommon complication after arthroscopy, but the conesquences can be severe if not diagnosed quickly. Arteriovenous fistula (AVF) is defined as an abnormal communication between an artery and a vein and can be con-

*Corresponding author. genital or acquired; in the latter case, AVFs are always pathological and must be quickly recognized. Their diagnosis can be difficult [9]. We report the case of a popliteal venous pseudoaneurysm secondary to AVF 6 months after arthroscopically assisted ACL reconstructtion, and a fibular AVF secondary to an orthopedic surgery.

\section{Case Report}

\subsection{Case Report 1}

A 33-year-old woman in good general health presented to our emergency department with increasing and invalidating pain in the left popliteal fossa. 6 months before she had undergone arthroscopically assisted ACL reconstruction of her left knee.

On first post-operatives controls, she did not show any vascular symptoms. The pulses were present, the lower limb was warm and she had a slight oedema due to orthopedic surgery. After 6 months she complained of some tension and discomfort at the level of the popliteal fossa.

On physical exam of the patient, the knee was stable in the frontal and sagittal plan, Lachmann's test was normal compared to the controlateral side and no anterior drawer was found. The surgical scars were almost invisible and 
no swelling or joint effusion could be found. The patient was afebrile and hemodynamically stable. All pulses were present with a normal neurological status. She presented with significant edema of her calf and foot as well as a tender, pulsatile mass measuring $3 \times 5 \mathrm{~cm}$ with audible bruit in her popliteal fossa. There were both a thrill and bruit over this mass. A bruit was audible over the peroneal artery and a thrill associated with the anterior tibial artery pulse at the ankle.

Initial investigation by venous duplex ultrasound scan showed no deep venous thrombosis and confirmed a left popliteal pseudoaneurysm lying to the popliteal artery and vein. There was no thrombus noted in the deep veins. Angiogram slighty CT scan showed an AVF arising from the left popliteal artery (Figure 1). An aneurysm measuring $3 \times 5 \mathrm{~cm}$ was seen to fill at the same time as the popliteal vein (Figure 2). Left femoral angiography showed an AVF starting from the popliteal artery (Figures 3(a) and (b)), while retrograde flow into anterior and posterior popliteal veins was seen at the time of filling of adjacent arteries.

Under general anesthesia, the patient underwent open primary repair of the traumatic AVF and aneurysm through a posterior approach. Surgical findings included AVF between popliteal artery and vein and a large venous pseudoaneurysm arising from the popliteal vein at the level of the arteriovenous communication (Figure 4). The popliteal vein pseudoaneurysm was directly repaired and a short saphenous vein graft was then harvested and used as a bypass with end-to-end anastomoses for the popliteal artery (Figure 5).

Immediate recovery was uneventful. Some left leg oedema developed, likely because of reperfusion effect.

\subsection{Case Report 2}

Two years after a serious polytraumatism, a 32-yearold patient presented a venous pseudoaneurysm and an

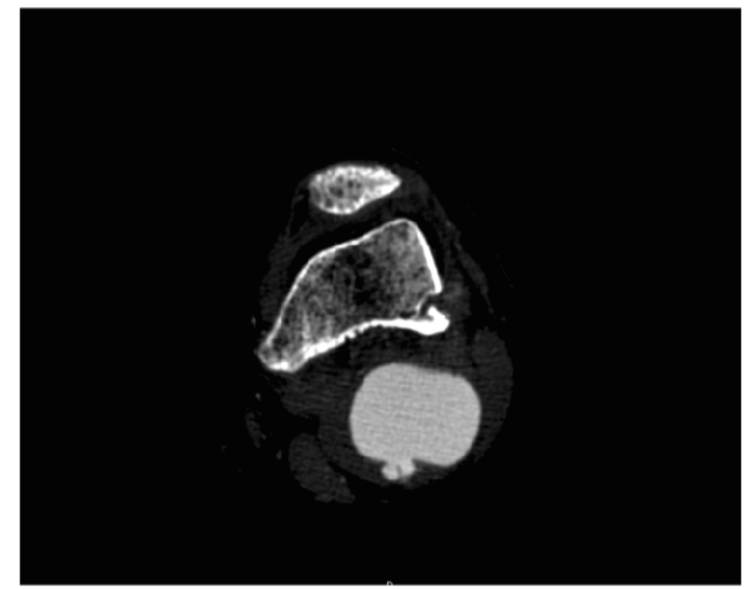

Figure 1. CT angiogram, demonstrating early popliteal venous filling with AVF.

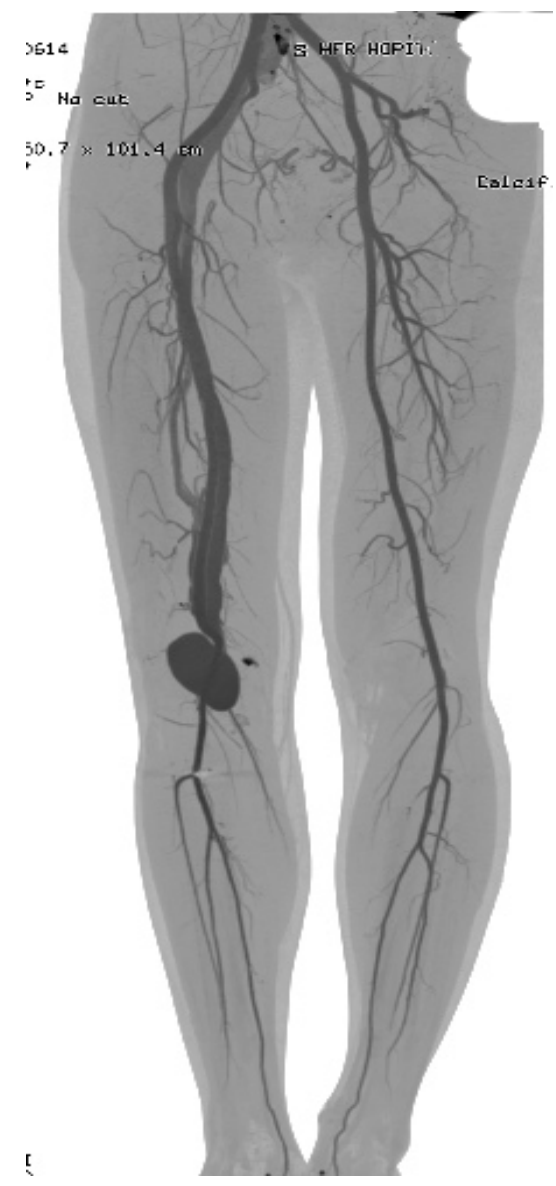

Figure 2. Angio-CT planar reconstruction, an early filling makes suspect a popliteal AVF, with a venous popliteal aneurysm.

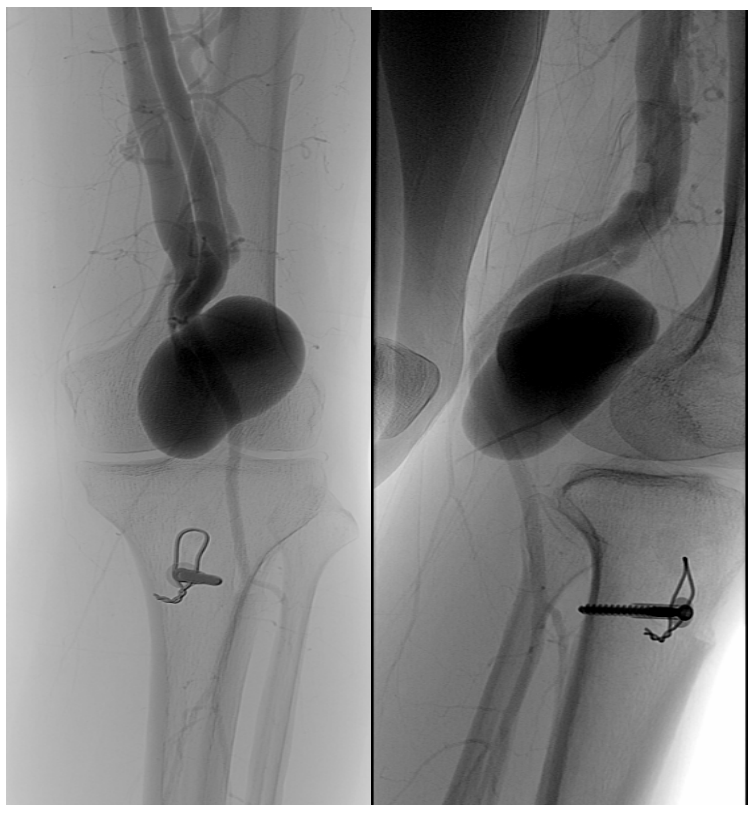

(a)

(b)

Figure 3. Angiogram, anteroposterior (a) and lateral (b) view of the AVF with popliteal vein pseudoaneurysm. 


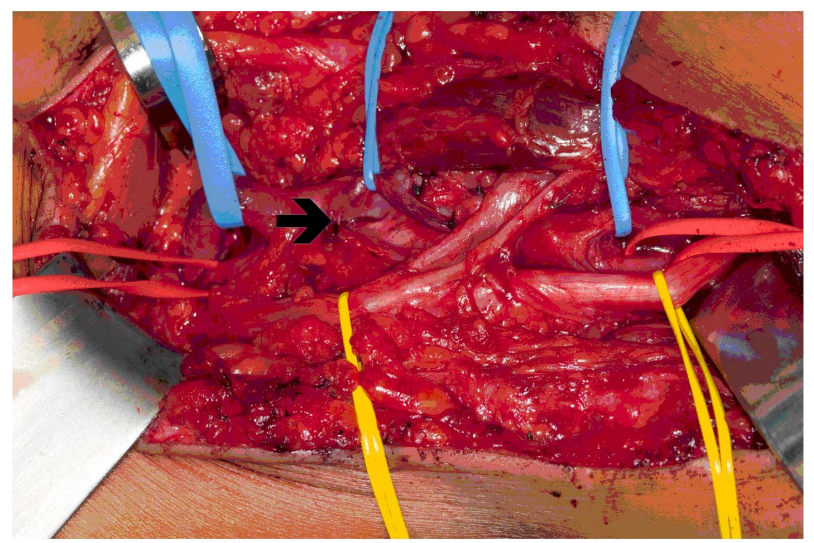

(a)

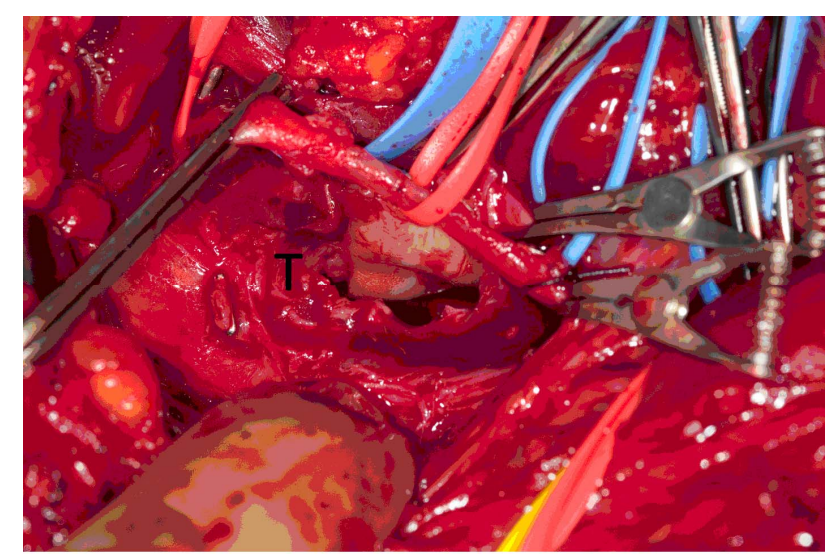

(b)

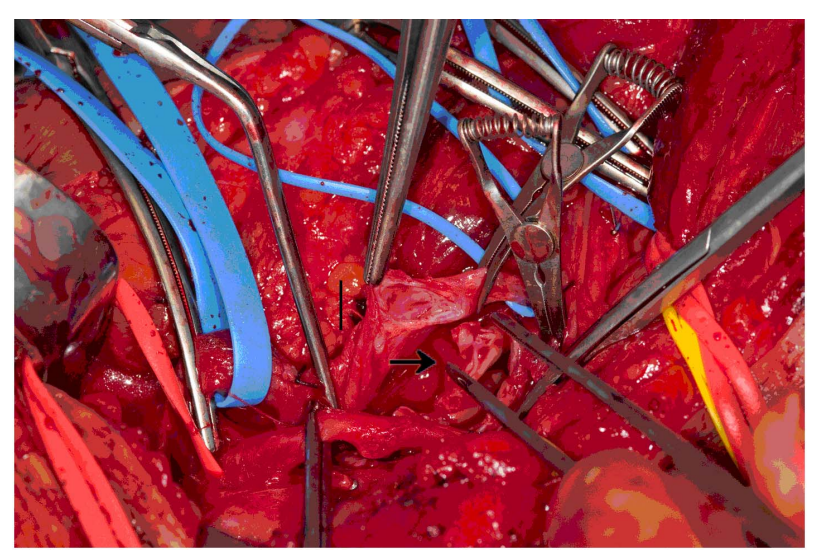

(c)

Figure 4. (a) Dilatation of the popliteal vein at the site of AVF $(\rightarrow)$.The vein is on the blue lacette, the popliteal artery on the red one and the tibial nerve on the yellow; (b) Fistula being resected $(T)$; (c) Popliteal artery $(\rightarrow)$ and vein $(\mid)$ separated after resection of the arteriovenous fistula.

arteriovenous fistula at the level of the fibular artery, half way on the calf. This young man had a comminuted open fracture of the lower limb, treated with external fixator and osteosynthesis plates. In the same time, a fracture of the femur with a third fragment had benefited from an osteosynthesis with centromedullar nailing. This fracture

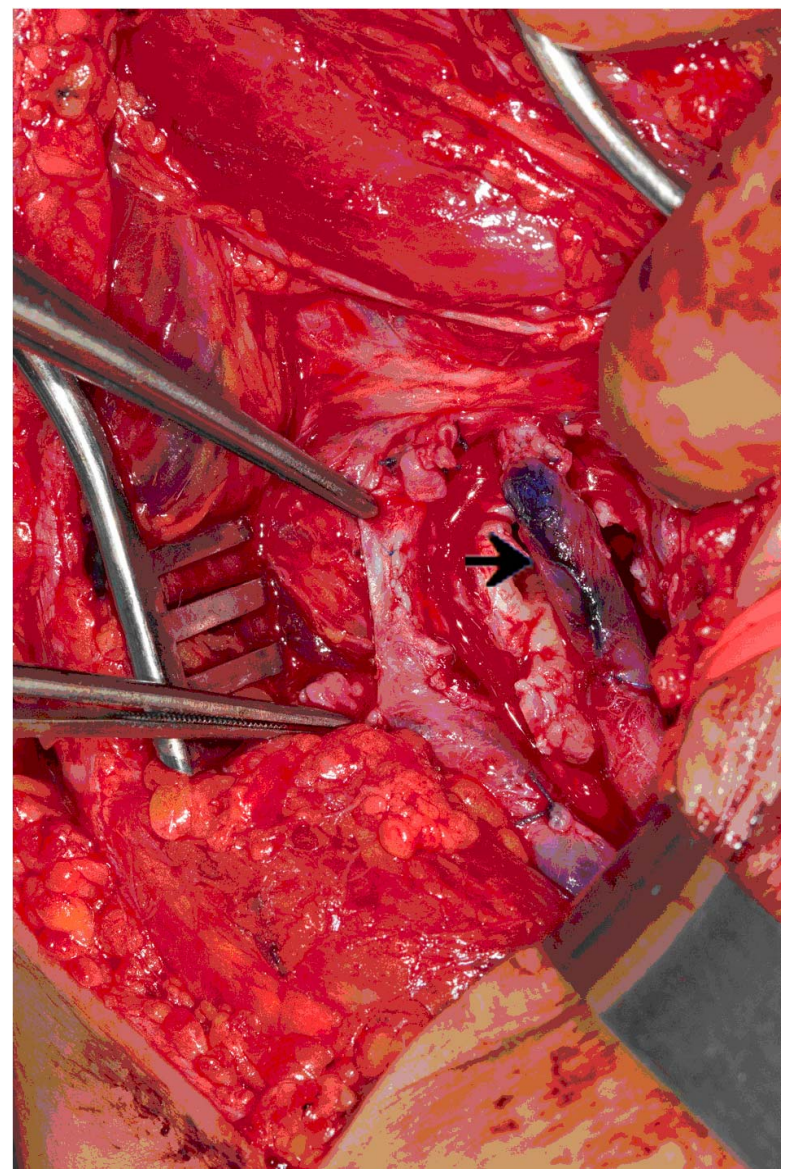

Figure 5. Popliteal vein pseudoaneurysm is directly repaired and we performed an end to end popliteal anastomosis bypass $(\rightarrow)$.

caused a shortening of the lower limb that was treated by surgery two years after the initial traumatism. During post-operative care following a persistent pain, a lateroexternal mass of the lower limb was discovered with a sensation of thrill. An echo-doppler confirmed the presence of a post-traumatic arteriovenous fistula, which was located at the level of the posterior tibial artery, half way on the calf with a $2.6 \mathrm{~cm}$-wide pseudoaneurysm.

The patient had an arteriography to visualize this fistula at best and discuss an endovascular treatment (Figure 6). In the absence of a neck it was impossible to perform an embolization. He was treated with an injection of thrombine in the aneurysm by way of the skin with echographic localization, and occlusion of the pedicule by endovascular way in the same time (Figure 7). The operation went well; it was followed by a thrombosis of the fibular artery and vein. The patient was under curative anticoagulant treatment for 6 weeks. The compression band was removed after 24 hours, and the patient was back home after 24 hours as well. In the followup, the patient was asymptomatic, he did not show any new complications and the ultrasound re- mained stable. 


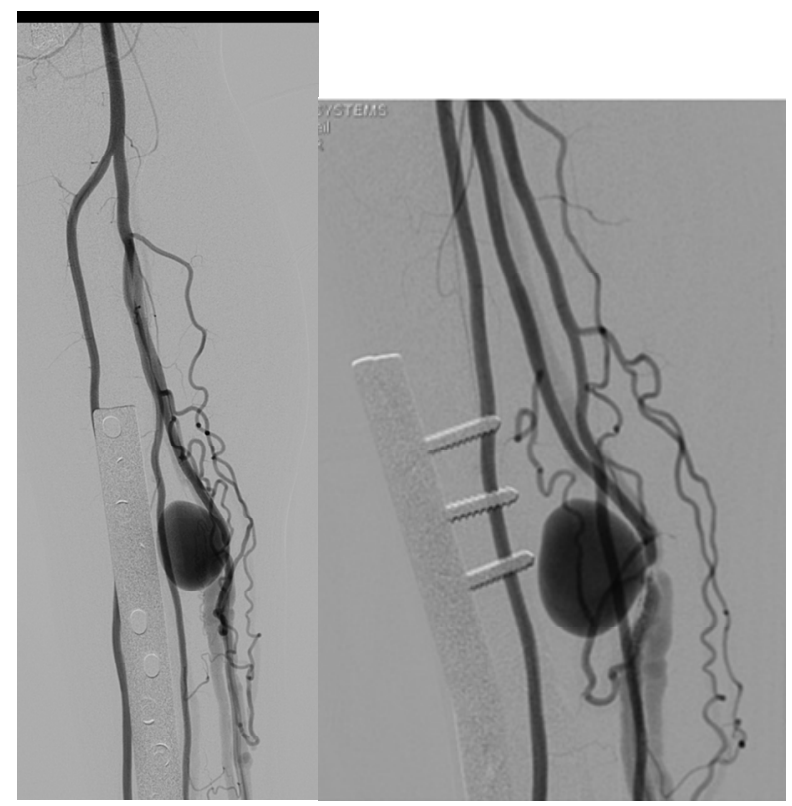

Figure 6. Angiogram, anteroposterior view of the AVF with vein pseudoaneurysm.

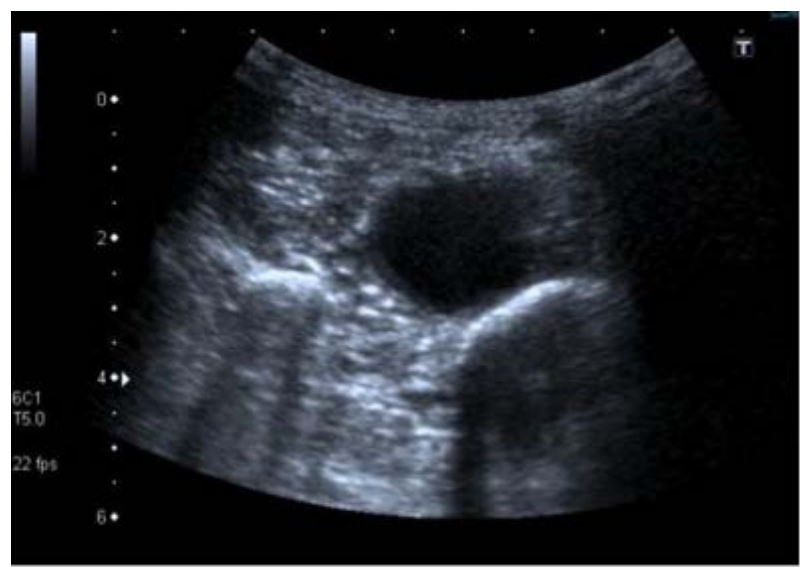

(a)

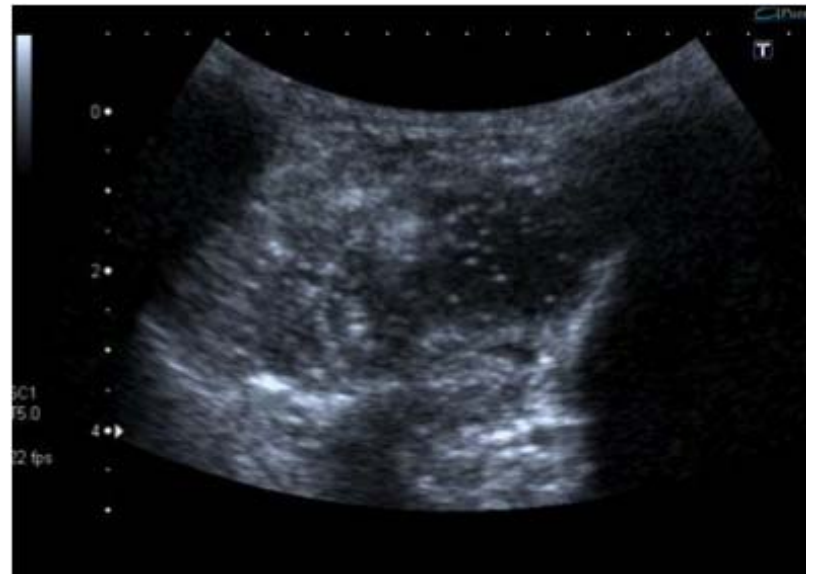

(b)

Figure 7. Ultrasound scan, (a) Before thrombin injection; (b) After thrombin injection.

\section{Discussion}

AVF arises as a result of combined injury of an artery and vein in close proximity [10]. Blood flow follows the path of least resistance, resulting in an endothelialized communication between the two vessels. Popliteal AVF may occur following penetrating trauma, either by missile or stab injuries or following blunt trauma associated with comminuted femur and proximal tibia fractures due to combined artery and vein laceration by bone fragments [11]. They can also be related to iatrogenic factors, such as surgical procedures, percutaneous arterial diagnostic or therapeutic intervention. Iatrogenic popliteal AVF has been reported following orthopaedic procedures including arthroscopy, arthrodesis and arthroplasty.

Vascular complications during orthopaedic surgery, although rare, do occur. Most vascular complications occur intraoperatively, immediately postoperatively or in the late postoperative period; they most commonly include lacerations, pseudoaneurysms, thrombosis, and arteriovenous fistulas. The operations most commonly associated with vascular injuries are knee arthroplasty, followed by hip arthroplasty, spinal surgery, and knee arthroscopy. Most commonly the popliteal artery is involved, followed by the tibial, superficial femoral, iliac, common and profunda femoral arteries. Color Doppler ultrasound is the initial imaging method used to evaluate a vascular injury.

Vascular injuries after arthroscopy of the knee are very rare, and several large series have reported an incidence of less than $0.005 \%$. Most vascular injuries involve the popliteal artery or vein, or both [12]. Despite the increasing number of arthroscopies performed annually and a greater exposure to them in orthopaedic training programs, occasional cases of vascular injury and arterial pseudoaneurysms after arthroscopic procedures in the knee [13-21] continue to be reported. Those in the knee may be due to the fact that the popliteal artery lies in very close vicinity to the posterior knee capsule and that internal rotation moves the vascular funicle relatively laterally, but external rotation stretches it across the attachment of the posterior horn of the medial meniscus, putting the vessel more at risk for iatrogenic injury.

The susceptibility of the popliteal artery and its branches to injury during arthroscopically assisted ACL reconstruction is due to several factors, including the surgical technique as well as the operator's experience [22]. The popliteal artery is close to the posterior capsule of the joint and it is moved forward during knee flexion. It can thus be injured during preparation of the femoral tunnel or by an unsecured K-wire. Visualization of this posterior region during knee arthroscopy is limited and possible injury is often not immediately recognized especially if a tourniquet is being used.

Traumatic AVF most commonly occur following pene- 
trating trauma, either by missile or stab injuries. AVF can also develop following blunt trauma because of combined artery and vein laceration by bone fragments [11].

Many factors play a role regarding the presentation and subsequent potential complications of AVF, like the nature of the trauma, the anatomic location (diameter of the artery and vein), the adequacy of the collateral circulation and the competence of the valves in the distal veins. Although some AVFs have a benign evolution with spontaneous closure, in most cases the fistulous opening enlarges which lead to degenerative changes in the arterial wall [23].

The effects of a fistula and how it is tolerated depend on two factors: the size and the duration of the shunt: Some fistulas can remain unnoticed and do not become apparent for several years [24]. Two clinical entities can be distinguished: an acute form and a chronic form resulting from an unrecognized or neglected fistula.

The classic signs of AVF (pulsatile swelling and a continuous thrill) are occasionally found in the acute phase, particularly in high-flow fistulas, but are not often looked for. Signs of arterial (pallor, coldness) or venous (edema, warmth, cyanosis, etc.) insufficiency, as well as pulsatile varicose veins, may also be present. The pulse may be absent, but is more often present but decreased [25].

Nevertheless, the formation of combined venous pseudoaneurysm and AVF after knee arthroscopy is extremely rare. Only one such case has been previously reported [10].

When a patient has popliteal swelling, mass, bruit, thrill, recurrent hemarthrosis, pain, calf edema after a knee arthroscopy, the possibility of a vascular injury should be considered early on in the diagnostic process. AVF can have serious consequences if not quickly diagnosed and treated. In a case series by Potter [18] the diagnosis of iatrogenic vascular injury was delayed in three of five patients. Complications in this group included massive bleeding, permanent foot drop, and above knee amputation. Unrecognized AVF may also result in severe complications such as high-output cardiac failure [26,27], pulmonary oedema [28], or chronic ischemic leg ulcer [29].

Once vascular injury is suspected, the diagnosis can be confirmed by arterial Color Doppler sonography followed by arteriography if doubt on the diagnosis persists. CT-scan or MRI may provide additional information regarding bone and soft tissue involment.

Small, asymptomatic AVF is usually not treated and followed until its spontaneous closure.

For many years, the treatment option available for iatrogenic popliteal AVF was surgical, through an open posterior approach that aimed for separation of the arterial and the venous system. Repair may be achieved by simple ligation or by disconnecting the vessels and re- pairing them by suturing the vessel walls, vein patch, or interposition graft. Nowadays endovascular treatment gain importance with better outcomes, because of material improving.

Endovascular and surgical procedures have both been described and proven effective in the treatment of iatrogenic AVF. Surgical interventions through an open posterior approach aim for separation of the arterial and the venous system. Repair may be achieved by simple ligation or by disconnecting the vessels and repairing them by suturing the vessel walls, vein patch, or interposition graft or endovascular stenting. Open repair is recommended to healthy and/or young patients and to patients whose lesions or anatomy is unsuitable for endovascular repair. In the first case an open procedure was selected, taking into account the young age of the patient and the fact that she was an athlete in excellent general health [27].

Endovascular techniques have been described and proven effective in the treatment of iatrogenic AVF, with the use of covered stent or metallic coils [30,31], glu and thrombin. It may play an even larger role in patients who are poor operative candidates or in reoperative procedures [1,32].

Regarding the second case, we chose this technique because of the patient's past medical history and because of the unfavourable localization of the fistula, and the possibility of compensation by the other arterial axes in case of thrombosis.

\section{Conclusions}

However degenerative arterial changes due to AVF are well described, reports on the presence of concomitant venous aneurysm are really scarced. Open surgery for iatrogenic AVF with venous aneurysm allows the ligation of the fistula and the reconstruction of the popliteal vein, without sign of venous thrombosis in the follow-up. AVF is an uncommon complication after arthroscopically assisted ACL reconstruction, but the consequences can be severe if not diagnosed quickly.

The initial injury causing AVF often results in subtle clinical signs, which may be easily overlooked. Importantly, the presence of peripheral pulses does not exclude arterial injury. Diagnosis and recent treatment reports have emphasized the importance of prompt diagnosis and treatment.

Endovascular treatment has proven its efficiency and should play a more important role in the future for the care of these patients.

\section{Acknowledgements}

The authors declared no conflicts of interest with respect to the authorship and/or publication of this article.

The authors received no financial support for the re- 
search and/or authorship of this article.

\section{REFERENCES}

[1] J. K. Nelms, G. L. Piper and J. C. Young, “Arteriovenous Fistula as a Rare Complication after Arthroscopic Meniscectomy,” The American Surgeon, Vol. 75, No. 5, 2009, pp. 432-434.

[2] N. C. Small, "Complications in Arthroscopic Surgery Performed by Experienced Arthroscopists," Arthroscopy, Vol. 4, No. 3, 1988, pp. 215-221. doi:10.1016/S0749-8063(88)80030-6

[3] O. H. Sherman, et al., "Arthroscopy-'No-Problem Surgery'. An Analysis of Complications in Two Thousand Six Hundred and Forty Cases," Journal of Bone and Joint Surgery (American Volume), Vol. 68, No. 2, 1986, pp. 256-265.

[4] R. P. Janssen, M. R. Scheltinga and H. A. Sala, "Pseudoaneurysm of the Popliteal Artery after Anterior Cruciate Ligament Reconstruction with Bicortical Tibial Screw Fixation," Arthroscopy: The Journal of Arthroscopic \& Related Surgery: Official Publication of the Arthroscopy Association of North America and the International Arthroscopy Association, Vol. 20, No. 1, 2004, pp. E4-E6.

[5] J. D. Evans, M. T. de Boer, P. Mayor, D. Rees and A. J. Guy, "Pseudoaneurysm of the Medial Inferior Genicular Artery Following Anterior Cruciate Ligament Reconstruction," Annals of the Royal College of Surgeons of England, Vol. 82, No. 3, 2000, pp. 182-184.

[6] M. Milankov, N. Miljkovic and M. Stankovic, "Pseudoaneurysm of the Medial Inferior Genicular Artery Following Anterior Cruciate Ligament Reconstruction with Hamstring Tendon Autograft,” The Knee, Vol. 13, No. 2, 2006, pp. 170-171. doi:10.1016/j.knee.2005.12.002

[7] J. K. Sekiya, B. C. Ong and J. P. Bradley, "Complications in Anterior Cruciate Ligament Surgery," The Orthopedic Clinics of North America, Vol. 34, No. 1, 2003, pp. 99105. doi:10.1016/S0030-5898(02)00058-5

[8] M. Kanko, et al., "Very Rare Aetiology of Giant Popliteal Pseudoaneurysm: Anterior Cruciate Ligament Surgery,” Postgraduate Medical Journal, Vol. 84, No. 989, 2008, pp. 158-159. doi:10.1136/pgmj.2007.062646

[9] F. Jimenez, et al., "Popliteal Artery and Venous Aneurysm as a Complication of Arthroscopic Meniscectomy," Journal of Trauma, Vol. 28, No. 9, 1988, pp. 1404-1405. doi:10.1097/00005373-198809000-00020

[10] R. Coleman, "Combined Arteriovenous Fistula and Venous Aneurysm Following Knee Arthrodesis,” ANZ Journal of Surgery, Vol. 76, No. 11, 2006, pp. 1030-1032. doi:10.1111/j.1445-2197.2006.03926.x

[11] G. Lebreton, et al., "Popliteal Arteriovenous Fistula Due to a Gunshot Injury,” Annals of Vascular Surgery, Vol. 24, No. 7, 2010, pp. e17-e21.

[12] D. J. Mullen and G. J. Jabaji, "Popliteal Pseudoaneurysm and Arteriovenous Fistula after Arthroscopic Meniscectomy," Arthroscopy, Vol. 17, No. 1, 2001, p. E1. doi:10.1053/jars.2001.16284

[13] D. E. Beck, J. G. Robison and J. W. Hallett Jr., "Popliteal
Artery Pseudoaneurysm Following Arthroscopy," The Journal of Trauma, Vol. 26, No. 1, 1986, pp. 87-89. doi:10.1097/00005373-198601000-00017

[14] M. Hilborn, et al., "Pseudoaneurysm after Therapeutic Knee Arthroscopy: Imaging Findings,” American Journal of Roentgenology, Vol. 163, No. 3, 1994, pp. 637-639. doi:10.2214/ajr.163.3.8079859

[15] J. T. Jeffries, et al., "Injury to the Popliteal Artery as a Complication of Arthroscopic Surgery. A Report of Two Cases," The Journal of Bone and Joint Surgery (American Volume), Vol. 69, No. 5, 1987, pp. 783-785.

[16] P. Vassallo, et al., "Popliteal Pseudoaneurysm and Arteriovenous Shunt Following Arthroscopic Meniscectomy: Case Report," Cardiovascular and Interventional Radiology, Vol. 12, No. 3, 1989, pp. 142-144. doi:10.1007/BF02577378

[17] F. Jimenez, et al., "Popliteal Artery and Venous Aneurysm as a Complication of Arthroscopic Meniscectomy," The Journal of Trauma, Vol. 28, No. 9, 1988, pp. 14041405. doi:10.1097/00005373-198809000-00020

[18] D. Potter and W. Morris-Jones, "Popliteal Artery Injury Complicating Arthroscopic Meniscectomy,” Arthroscopy: The Journal of Arthroscopic \& Related Surgery: Official Publication of the Arthroscopy Association of North America and the International Arthroscopy Association, Vol. 11, No. 6, 1995, pp. 723-726.

[19] M. P. Manning and J. H. Marshall, “Aneurysm after Arthroscopy,” The Journal of Bone and Joint Surgery, British Volume, Vol. 69, No. 1, 1987, p. 151.

[20] R. J. Guy, T. J. Spalding and L. J. Jarvis, "Pseudoaneurysm after Arthroscopy of the Knee. A Case Report," Clinical Orthopaedics and Related Research, No. 295, 1993, pp. 214-217.

[21] E. A. Sarrosa and D. J. Ogilvie-Harris, "Pseudoaneurysm as a Complication of Knee Arthroscopy," Arthroscopy: The Journal of Arthroscopic \& Related Surgery: Official Publication of the Arthroscopy Association of North America and the International Arthroscopy Association, Vol. 13, No. 5, 1997, pp. 644-645.

[22] J. Puig, et al., "Transarterial Embolization of an Inferior Genicular Artery Pseudoaneurysm with Arteriovenous Fistula after Arthroscopy," Korean Journal of Radiology, Vol. 8, No. 2, 2007, pp. 173-175. doi:10.3348/kjr.2007.8.2.173

[23] D. S. Summer, "Hemodynamics and Pathophysiology of Arteriovenous Fistulae,” In: R. B. Rutherford, Ed., Vascular Surgery, 5th Edition, Saunders, Philadelphia, 2000, pp. 1400-1425.

[24] W. Huang, J. L. Villavicencio and N. M. Rich, "Delayed Treatment and Late Complications of a Traumatic Arteriovenous Fistula,” Journal of Vascular Surgery: Official Publication, the Society for Vascular Surgery and International Society for Cardiovascular Surgery, North American Chapter, Vol. 41, No. 4, 2005, pp. 715-717.

[25] J. B. Carr and K. A. Jansson, "An Unusual Case of Vascular Dysplasia Related to Knee Arthroscopy,” Arthroscopy: The Journal of Arthroscopic \& Related Surgery: Official Publication of the Arthroscopy Association of North America and the International Arthroscopy Asso- 
ciation, Vol. 17, No. 2, 2001, pp. 196-199.

[26] J. H. Kim, et al., "Endovascular Treatment of an Iatrogenic Large Vessel Arteriovenous Fistula Presenting as High Output Heart Failure: A Case Report,” Vascular and Endovascular Surgery, Vol. 46, No. 6, 2012, pp. 495-498. doi:10.1177/1538574412452158

[27] A. Kuehnl, et al., "Young Girl Presenting with Heart Failure 5 Years after Laparoscopic Appendectomy. Case Report of an Ilio-Iliac AV Fistula," European Journal of Vascular and Endovascular Surgery: The Official Journal of the European Society for Vascular Surgery, Vol. 40, No. 1, 2010, pp. 107-109.

[28] J. Malik, et al., “Arteriovenous Fistula after Femoral Artery Puncture Leading to Pulmonary Edema: The Role of Ultrasonography,” Prague Medical Report, Vol. 113, No. 1, 2012, pp. 49-52.

[29] S. Suknaic, et al., "Chronic Ischaemic Leg Ulcer as a Late Complication of Post-Traumatic Arteriovenous Fistula,”
Phlebology, Vol. 27, No. 3, 2012, pp. 124-127. doi:10.1258/phleb.2011.011018

[30] B. E. Cil, et al., "Iatrogenic Femoral Arteriovenous Fistula: Endovascular Treatment with Covered Stent Implantation and 4-Year Follow-Up,” Diagnostic and Interventional Radiology, Vol. 12, No. 1, 2006, pp. 50-52.

[31] M. L. Marin, et al., "Transfemoral Endoluminal Stented Graft Repair of a Popliteal Artery Aneurysm,” Journal of Vascular Surgery: Official Publication, the Society for Vascular Surgery and International Society for Cardiovascular Surgery, North American Chapter, Vol. 19, No. 4, 1994, pp. 754-757.

[32] Y. N. Park, et al., "Endovascular Therapy of a Traumatic Chronic Popliteal Arteriovenous Fistula Using an AMPLATZER Vascular Plug," Journal of Vascular and Interventional Radiology, Vol. 21, No. 11, 2010, pp. 17791782. doi:10.1016/j.jvir.2010.07.023 